\title{
The Neural Correlates of Social Anxiety Disorder and Response to Pharmacotherapy
}

\author{
Clinton D Kilts*, I,2, Jeffrey E Kelsey', Bettina Knight', Timothy D Ely', F DuBois Bowman³, Robin E Gross', \\ Amy Selvig', Angelita Gordon ${ }^{3}$, D Jeffrey Newport' and Charles B Nemeroff' \\ 'Department of Psychiatry and Behavioral Sciences, Emory University School of Medicine, Atlanta, GA, USA; ${ }^{2}$ Emory Center for Positron Emission \\ Tomography, Emory University School of Medicine, Atlanta, GA, USA and ${ }^{3}$ Department of Biostatistics, Emory University School of Public Health, \\ Atlanta, GA, USA
}

\begin{abstract}
This study attempted to define further the neural processing events underlying social anxiety in patients with social anxiety disorder $(\mathrm{SAD})$ and their response to pharmacotherapy. Social anxiety-related changes in regional cerebral blood flow were defined by $\left[{ }^{15} \mathrm{O}\right] \mathrm{H}_{2}$ positron emission tomography (PET) in medication-free individuals with generalized SAD (GSAD), and age- and sex-matched comparison subjects, and analyzed using a linear mixed effects model. PET studies were again acquired in the gSAD individuals following an 8-week, flexible dose treatment trial of nefazodone. Both script-guided mental imagery of an anxiogenic social situation and a confrontational mental arithmetic task were associated with marked increases in self-rated anxiety in both subject groups. For gSAD subjects, social anxiety induced by guided mental imagery was associated with increased activity in the left postcentral gyrus and lenticulate, and the right inferior frontal and middle temporal gyri. Social anxiety induced by the mental arithmetic task was associated with activation of the medial and left dorsolateral prefrontal cortex, cerebellum, thalamus, insula, and ventral striatum. Both tasks were associated with relative decreases in activity in the right amygdala and the hippocampus. A direct group comparison indicated that comparison subjects exhibited a differing pattern of social anxiety-related neural activations. Nefazodone treatment was associated with marked clinical improvement. Comparison of social anxiety-related neural activations prior to and after nefazodone administration indicated greater activity in the precentral gyrus, insula, midbrain/hypothalamus, and middle frontal and anterior cingulate gyrus prior to treatment, and greater activity in the left middle occipital and bilateral lingual gyri, postcentral gyrus, gyrus rectus, and hippocampus after treatment. The results of an analysis relating neural activity and treatment-related changes in symptom severity indicated differential neural responses associated with states of symptom remission vs partial response. The observed social anxiety-related changes in distributed neural activity are consistent with cognitive models of SAD and adaptive decreases in amygdala activity in response to social anxiogenics, and support the association of altered frontal cortical responses with treatment response.

Neuropsychopharmacology (2006) 31, 2243-2253. doi: I 0.1038/sj.npp. I 30 I053; published online 8 March 2006
\end{abstract}

Keywords: social anxiety disorder; social phobia; positron emission tomography (PET); regional cerebral blood flow (rCBF); nefazodone; amygdala

\section{INTRODUCTION}

Social anxiety disorder (SAD), often referred to as social phobia, is characterized by fear and avoidance of social situations associated with being observed or evaluated by others or a fear of embarrassing oneself. Epidemiological surveys indicate that SAD represents one of the most common psychiatric disorders with 12-month and lifetime

*Correspondence: Dr CD Kilts, Department of Psychiatry and Behavioral Sciences, Emory University School of Medicine, 101 Woodruff Circle, Suite 4000, Atlanta, GA 30322, USA, Tel: + I 404 727 8262, Fax: + I 404727 3233, E-mail: sdpcdk@emory.edu Received 6 June 2005; revised I December 2005; accepted 24 January 2006

Online publication: 30 January 2006 at http://www.acnp.org/citations/ Npp0 I 3006050362/default.pdf prevalence rates of 6.8 and $12.1 \%$, respectively (Kessler $\mathrm{et} \mathrm{al}$, 2005a,b). Individuals with SAD experience a markedly diminished quality of life due to their reduced ability to initiate and maintain friendships and marriages, to stay in school, to seek career advances, and a reduced sense of well being (Schneier et al, 1994; Stein and Kean, 2000; Safren et al, 1997; Wittchen et al, 2000; Katzelnick et al, 2001). In spite of the fact that SAD is associated with severe impairments in social, occupational and family functioning, it has been widely neglected by affected individuals and health-care professionals (Katzelnick et al, 2001; Liebowitz et al, 1985; Weiller et al, 1996). Recent studies estimate that only $5 \%$ or less of individuals with SAD seek help from a mental health professional (Schneier et al, 1992; Davidson et al, 1993). SAD has a typical age of onset in early adolescence and affects slightly more women than men 
(Chartier et al, 1998). The high rate of comorbid psychiatric disorders that usually occur after the onset of SAD suggests that SAD may be a risk factor for other psychiatric disorders including substance abuse (Katzelnick et al, 2001; Weiller et al, 1996). SAD therefore represents a major public health problem due to its prevalence, morbidity, role as a risk factor, and chronic and neglected nature.

$\mathrm{SAD}$ is frequently thought of as a nongeneralized fear and avoidance of specific social activities such as public speaking. Indeed, this is frequently the most symptomprovoking social situation in nongeneralized SAD (Stein et al, 1996). However, the increasing recognition that a more prevalent and impaired subgroup of individuals with SAD fear and avoid a wide array of social situations has led to the recognition of generalized SAD (gSAD) (den Boer, 1997; Kessler et al, 1998). The neurobiology of SAD remains poorly understood (Bell et al, 1999), though conceptual frameworks neeeded to guide such investigations have recently been proposed (Mathew et al, 2001). Functional brain imaging studies of induced anxiety states in healthy volunteers (Kimbrell et al, 1999) or in patients with other anxiety disorders including simple phobia (Rauch et al, 1995), obsessive-compulsive disorder (Breiter et al, 1996; Saxena et al, 1999), or posttraumatic stress disorder (Shin et al, 1999; Birbaumer et al, 1998; Britton et al, 2005) implicate altered activity in limbic (amygdala), paralimbic (insula, cingulate, and orbitofrontal cortex), and striatal (caudate nucleus, lenticulate) structures. Recent brain imaging studies of SAD have explored the neural response to facial expressions of emotion and to symptom provocation paradigms, and disorder-related abnormalities in dopamine neurotransmission. Functional magnetic resonance studies of SAD implicate an exaggerated response of the amygdala related to the perception of fear (Birbaumer et al, 1998), anger or contempt (Stein et al, 2002), and in learned fear responses (Schneider et al, 1999). Positron emission tomography (PET) imaging studies of symptom provocation in individuals with $\mathrm{SAD}$ indicate that anxiety related to the anticipation or performance of a public speaking task was associated with activation of the amygdala area, and dorsolateral prefrontal and inferior temporal cortex (Tillfors et al, 2001; Tillfors et al, 2002). A recent PET study (Furmark et al, 2002) noted a decreased response to public speaking in the amygdala, hippocampus, and adjacent temporal cortex following either cognitive behavioral therapy or citalopram administration. PET studies of SAD also indicate a reduced density of striatal $\mathrm{D}_{2}$ dopamine receptors (Schneier et al, 2000) and dopamine transporters (Tiihonen et al, 1997) that support a model of dopamine dysregulation (Mathew et al, 2001).

Prominent cognitive behavioral models of SAD postulate the tendency towards heightened self awareness and monitoring as a response to a fear of being observed and evaluated by others (Rapee and Heimberg, 1997). The personality dimension of self-criticism is robustly associated with SAD (Cox et al, 2004). The experimental challenge of inducing social anxiety in a clinical population characterized by the avoidance of such situations was addressed by assessing convergent neural responses to both the script-guided mental imagery of personal social situations associated with anxiety and to a social performance and evaluation task (ie confrontational mental arithmetic). $\left[{ }^{15} \mathrm{O}\right] \mathrm{H}_{2}$ PET studies were used to test the hypothesis that social anxiety states associated with gSAD reflect alterations in neural information processing in brain areas related to social cognition (eg orbitofrontal cortex, superior temporal sulcus, and amygdala) (Brothers, 1990; Adolphs, 2001) and areas related to self-reference (eg, medial prefrontal cortex, posterior cingulate gyrus) (Kelley et al, 2002). Neural activations related to induced social fear and anxiety were reassessed following an 8-week, flexible dose trial of nefazodone administration to identify the neural correlates of treatment response. The neocortical response to treatment was assessed to explore treatment effects on the neural representations of gSAD-related self-representations (Abbott and Rapee, 2004) and of cognitive reappraisal mechanisms involved in regulating emotional reactions (Ochsner et al, 2002).

\section{PATIENTS AND METHODS}

\section{Study Participants}

Twelve right-handed patients (seven men and five women; $38 \pm 8.1$ ( $1 \mathrm{SD}$ ) years of age) with gSAD (illness duration of $28.8 \pm 10.4$ years) participated in this study. The diagnosis of gSAD was made according to DSM-IV criteria and confirmed by a structured interview using the Structured Clinical Interview for DSM-IV (SCID) (First et al, 2002). Patients endorsed marked symptoms of SAD as assessed using the 24-item Leibowitz Social Anxiety Scale (LSAS) (Liebowitz, 1987) with a total score of $85.8 \pm 18.8$ and the absence of symptoms of major depression was documented by a score of $8.3 \pm 2.8$ using the 17-item Hamilton Rating Scale for Depression (Hamilton, 1960). Subject symptoms were further characterized by the rated intensity (0-3) of fear or anxiety and avoidance associated with specific social situations using the subscales of the LSAS. General mental imagery ability was assessed using the 35-item Questionnaire on Mental Imagery (QMI) (Sheehan, 1967). Study exclusion criteria included the presence of other Axis I or II disorders, a history of drug dependence or abuse, severe head injury, neurologic disorders, pregnancy, or the prescribed use of psychotropic medications. Participating subjects were either medication naive (four individuals) or medicationfree for one week (one individual) or longer (seven individuals: $38.1 \pm 26$ months, range 3-96 months) prior to baseline PET imaging and subsequent treatment with nefazodone. The daily dose of nefazodone was individually adjusted based on weekly assessments of symptom response and occurrence of side effects with an average daily dose at the 8 -week end point of $477 \pm 98 \mathrm{mg}$ (range 300-600 mg).

Six right-handed healthy volunteers (four men and two women; $32.4 \pm 5.8$ ( $1 \mathrm{SD}$ ) years of age) were studied as a comparison group. Absence of any psychiatric disorder was verified using the SCID. The research protocol was approved by the Emory University Investigational Review Board. Written informed consent was obtained after the procedures comprising the research protocol had been fully explained. 


\section{PET Image Acquisitions}

For both subject groups, $\left[{ }^{15} \mathrm{O}\right] \mathrm{H}_{2}$ PET images were acquired during a resting state (eyes closed, ears unoccluded), following a confrontational mental arithmetic task, and during each of two states induced by script-guided mental imagery. A control nonsocial imagery condition involved the mental imagery of a beach or forest scene absent of other people that was guided by a standardized script (Bourne, 1995). A social anxiety imagery condition involved the script-guided imagery of the individual-specific social situation associated with maximal fear and avoidance. Scripts were constructed using a modified version of the Vietnam Stressful Scene Construction Questionnaire (Pitman et al, 1987). Imagery scripts defining the social situation were assembled from the elicited bodily sensations (eg heart racing) selected from a 35 -item checklist and a hand-written narrative describing the environmental context of the situation. Individual scripts were audio taped in the first person, present tense and edited to a standard length of approximately $60 \mathrm{~s}$. The confrontational mental arithmetic task from the Trier Social Stress Test (Kirschbaum et al, 1993) was used as a generic anxiogenic condition. It involved an unfamiliar investigator and subjects were instructed to sequentially subtract 13 from the number 2083 for the first trial, and from 1654 for the second trial. The investigator urged the subjects to 'go faster' and any error was emphasized and resulted in an instruction to start over again at 2083 or 1654 . This task was approximately $60 \mathrm{~s}$ in duration.

Task-related regional cerebral blood flow ( $\mathrm{rCBF}$ ) was determined with the Siemens ECAT 951 PET scanner (31 contiguous planes spaced at $3.375 \mathrm{~mm}$ intervals) following the bolus intravenous infusion of $35 \mathrm{mCi}$ of $\left[{ }^{15} \mathrm{O}\right] \mathrm{H}_{2}$ (Mazziotta et al, 1985). Each participant was scanned eight times in an imaging session, twice in each of the four conditions of rest, control script imagery, social anxiety script imagery, and mental arithmetic. This same order of task conditions was repeated in a fixed order design. For the two script imagery conditions, scripts were presented binaurally with instructions to first listen to the script and then mentally re-enact the scene described. $\left[{ }^{15} \mathrm{O}\right] \mathrm{H}_{2}$ administration was coincident with the end of each script and 90-s single frame studies were initiated by the detection of cerebral radioactivity and acquired in a 2-D mode. For the confrontational mental arithmetic task condition, the $\left[{ }^{15} \mathrm{O}\right] \mathrm{H}_{2}$ dose was administered coincident with the termination of the task to avoid neural activations related to computation, verbalization, or attention. Therefore, image acquisitions were devoid of neural responses to task requirements (auditory stimulation, mathematical computation, response verbalization) and focused on the task responses. $\left[{ }^{15} \mathrm{O}\right] \mathrm{H}_{2}$ injections were made at 10 -min intervals.

The subjective responses to tasks were self-assessed using 0 - to 10-point analog scales following offset of the scanner. Using these scales, participants self-rated the experience of anxiety, sadness, anger, happiness, and relaxation for all conditions, as well as the vividness of the mental image for the imagery tasks. The scale anchor points for each selfassessment were 'not at all, none' for 0 and 'a great deal' for 10 .

\section{PET Image Postprocessing and Data Analysis}

Images were reconstructed using a measured attenuation correction $\left({ }^{68} \mathrm{Ge} /{ }^{68} \mathrm{Ga}\right.$ transmission scans). PET image postprocessing was conducted using SPM99 (Wellcome Department of Cognitive Neurology, http://.fil.ion.ucl.ac.uk/ spm). PET images for each subject were aligned and resliced (Woods et $a l, 1998 \mathrm{a}$ ) and then spatially normalized to a population-representative PET atlas (Woods et al, 1998b) centered in Talairach stereotaxic coordinates (Talairach and Tournoux, 1988). The PET images were then smoothed by Gaussian filtering to a final resolution of $9 \mathrm{~mm}$ FWHM and normalized for global blood flow by proportionate scaling.

Correlation exists between localized rCBF measurements from the multiple scans across conditions obtained for each individual subject. In addition, the variability of localized measures of rCBF differs across experimental conditions and treatment periods. These data characteristics prompted the use of a general linear mixed effects model for statistical analysis (Verbeke and Molenberghs, 2000), rather than a general linear model (GLM) (Neter et al, 1996) approach that assumes independence and equal variance across all scans. We conducted a voxel-by-voxel mixed model analysis (Bowman and Kilts, 2003) to estimate rCBF means corresponding to conditions preceding and following an 8 -week regimen of nefazodone administration. The model included subject-specific random effects for experimental conditions (rest, social anxiety imagery, neutral imagery, mental arithmetic) and treatment period (pretreatment or post-treatment). By modeling unstructured covariance matrices for both random effects, this model (Bowman and Kilts, 2003) incorporated correlations between an individuals repeated scans and permitted heterogeneity across study conditions.

To address the study objectives, secondary parameters (contrasts), corresponding $t$-statistic images, and statistically significant $(p$-value $<0.001)$ activation maps were generated. Pretreatment contrasts were calculated to define the neural correlates of social anxiety by comparing mean rCBF across the two social anxiety provocation tasks to both the rest and neutral imagery control conditions. The impact of nefazodone treatment on social anxiety-related brain activity was examined by defining task-specific mean changes in rCBF from the pre- to post-treatment periods. Our examination of treatment impact focused on the social anxiety imagery task as well as on the rest condition, since we expected the subjects with gSAD to experience social anxiety at rest related to being in the PET scanner and awareness of being observed. All contrasts not involving the pretreatment rest condition included statistical adjustments for the level of anxiety observed during the initial rest condition (Figure 1). Specifically, we estimated the effect for each condition, relative to the pretreatment rest condition, and then employed these adjusted estimates to compute the contrasts of interest.

To estimate the relationship between changes in $\mathrm{rCBF}$ and differing treatment-related changes in symptom severity (as measured by the LSAS), we fit a model containing effects for each of the four conditions and an effect for the LSAS score (standardized by mean centering), with all effects stratified by treatment period. Testing linear combinations of the model parameters allowed the localization of significant 

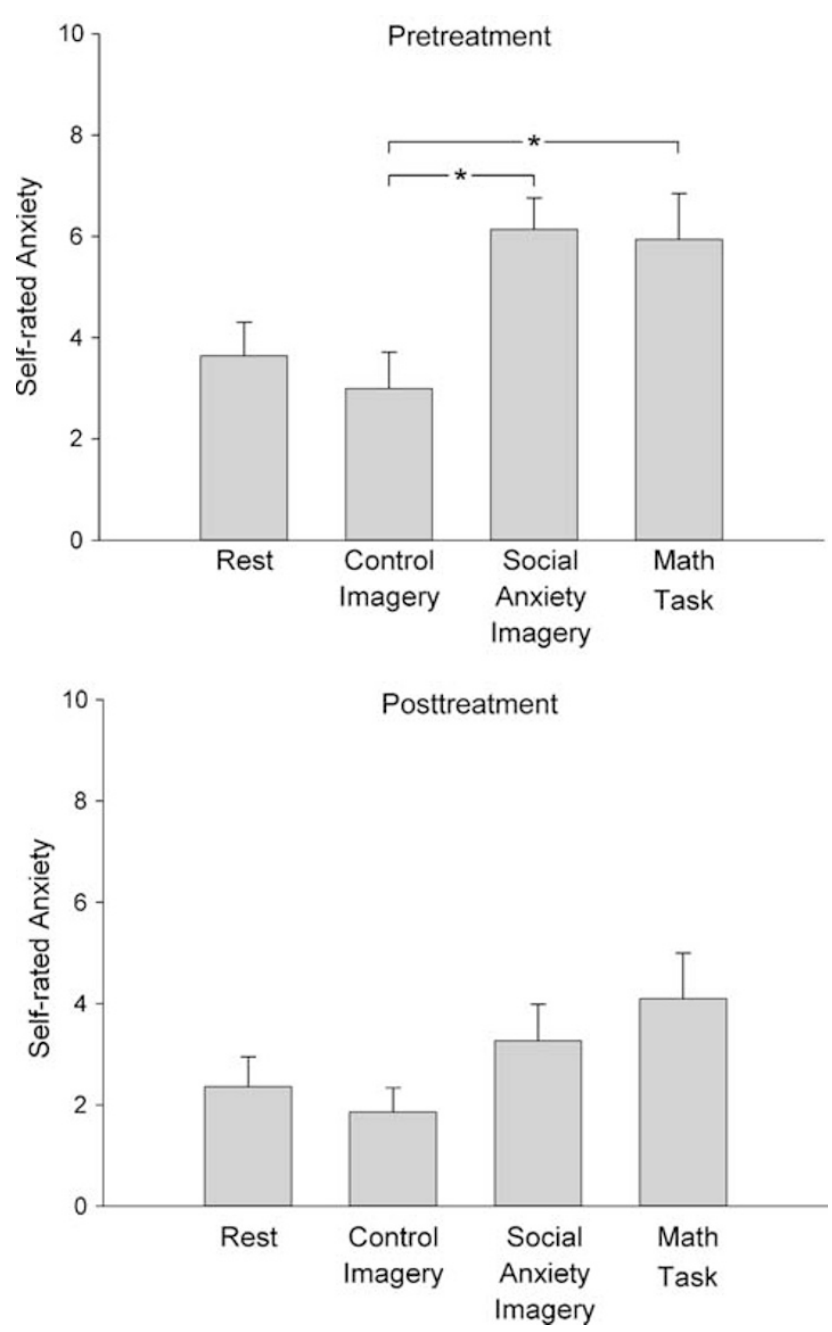

Figure I Anxiety response to the rest, control mental imagery, social anxiety imagery, and math task conditions prior to and following an 8-week course of treatment with nefazodone in a sample of individuals with gSAD. Asterisks indicate significant $(p<0.05)$ differences between conditions.

changes in brain activity associated with varying nefazodone-related reductions in symptoms of gSAD. Specifically, we identified brain areas that exhibited significant $(p<0.005)$ changes in social anxiety-related activity corresponding to symptom remission and partial response by defining secondary parameters with a pretreatment LSAS score of 90 and post-treatment LSAS scores of 20 and 45 , respectively. For stable estimation and reliable inferences, we only specified LSAS scores in our contrasts that were within the range of the data collected for our sample.

\section{RESULTS}

\section{Subjective Response to Anxiogenic Tasks}

Subjects with gSAD experienced mild to moderate anxiety during the resting state that decreased with subsequent mental imagery of the nonsocial control scene (Figure 1). Mental imagery of individualized scripts of social situations associated with social anxiety was associated with mild to intense anxiety for medication-free individuals with gSAD (Figure 1; mean anxiety self-rating of $6.3, \mathrm{SD}=1.2, N=11$ ). A one-way ANOVA of averaged data demonstrated a main effect of condition on self-rated anxiety $\left(\mathrm{F}_{3,37}=5.0\right.$, $p=0.005$ ), while post hoc analysis (Tukey's method) revealed significant $(p<0.05)$ increases in anxiety, compared to the nonsocial control imagery condition, for both the social anxiety imagery and confrontational mental arithmetic task (Figure 1, mean of 6.0, $\mathrm{SD}=1.7, N=8$ ) conditions. Comparison between trials supported the within-subject reproducibility of script-guided imagery-induced social anxiety. The reassessment of anxiety responses following an 8-week course of treatment with nefazodone indicated a reduction in the anxiety response across all conditions (Figure 1). A one-way ANOVA of averaged data indicated no effect of condition on self-rated anxiety after nefazodone treatment $(p=0.12)$.

For the comparison subjects, a one-way ANOVA of averaged data demonstrated a main effect of condition on self-rated anxiety $\left(\mathrm{F}_{3,16}=5.6, p=0.008\right)$, while post hoc analysis (Tukey's method) revealed significant $(p=0.008)$ increases in anxiety, compared to the nonsocial control scene imagery condition, for the social anxiety imagery, but not confrontational mental arithmetic, condition $(p=0.07)$. The gSAD and comparison subject samples did not differ in the magnitude of the self-rated anxiety response to either the social anxiety imagery $(p=0.77)$ or confrontational mental arithmetic $(p=0.56)$ tasks, when controlling for group differences in baseline anxiety observed in the rest condition.

\section{PET Imaging}

Neural activations related to social anxiety. Relative to the nonsocial imagery control condition, script-guided mental imagery of an anxiogenic social situation by individuals with gSAD was associated with relative increased activity in the left postcentral gyrus and putamen, and the right inferior frontal and middle temporal gyri, and relative decreases in activity in the right middle temporal gyrus, left precuneus, posterior cingulate gyrus, right caudate nucleus, and left hippocampus and right amygdala (Table 1, Figure 2). The right amygdala response was negatively correlated with the left ventrolateral prefrontal cortex response (Figure 5). Comparison subjects exhibited a distinct pattern of neural activations related to mental reenactment of a social anxiety-related situation that included the right lateral orbital frontal cortex and temporal pole, occipitotemporal cortex, ventral striatum, right posterior cingulate and medial prefrontal cortex, superior temporal sulcus, and insula (Table 2).

Owing to a change in experimental design after the onset of the study, eight of the 12 subjects with gSAD participated in the confrontational mental arithmetic task. In these subjects social performance and evaluation related to the confrontational mental arithmetic task was associated, relative to the nonsocial imagery control condition, with increased activity in the medial and left superior prefrontal cortex, cerebellum, thalamus, insula, and ventral striatum; relative decreases in activity were observed in the visual and dorsal cingulate cortex, and limbic areas including the hippocampus and amygdala (Table 1, Figure 2). Compar- 
Table I Anatomical and Stereotaxic Location of Differential Neural Activations Related to Re-Experiencing Social Anxiety-Provoking Situations (Left) or Social Performance and Evaluation (Right) for Subjects with Generalized Social Anxiety Disorder

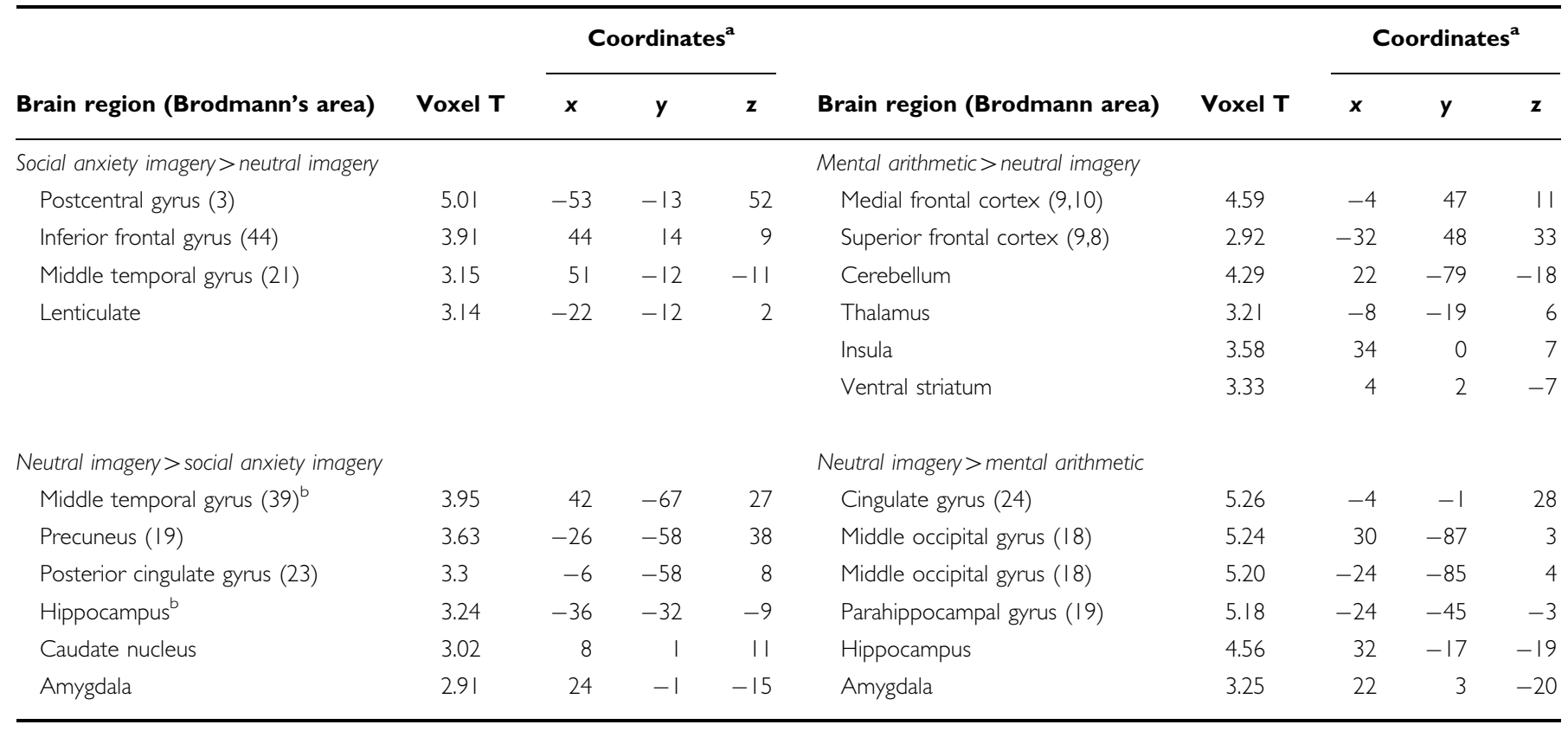

avalues represent the stereotaxic location of voxel maxima or minima with coordinates in Talairach space in millimeters lateral to midline $(x)$, anterior/posterior to the anterior commissure $(y)$, and superior/inferior to the commissural line (z). The t statistics were uncorrected for multiple comparisons.

${ }^{b}$ Greater relative decrease in activity associated with imagery-induced social anxiety in GSAD vs healthy comparison subjects.

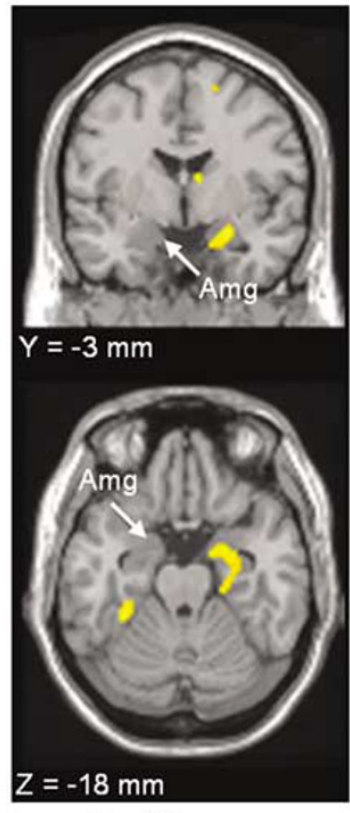

Social Imagery

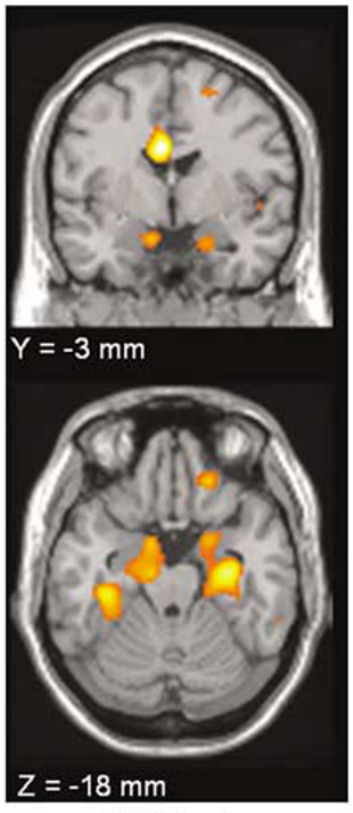

Math Task
Figure 2 Relative decreases in rCBF in the amygdala and hippocampus associated with symptom provocation in individuals with generalized social anxiety disorder. Response to guided mental imagery of socially anxious situations (left) and social performance and evaluation related to a confrontational mental arithmetic task (right) are illustrated in coronal (top) and axial (bottom) images. Difference images represent comparisons to a common mental imagery control condition and significant activations were $p<0.00 \mathrm{I}$, uncorrected. Abbreviations refer to the amygdala (Amg).

ison subjects exhibited a distinct pattern of neural activations related to the psychosocial stress posed by the mental arithmetic task that included the bilateral posterior
STS, thalamus, precuneus, and the inferior, medial, and middle frontal gyri (Table 2).

A direct contrast of the gSAD and healthy comparison subjects groups indicated that social anxiety in comparison subjects related to the social-neutral imagery condition comparison was associated with greater activity for the right fusiform (BA 18/19; $x=38, y=-68, z=-10 \mathrm{~mm}$ ) and lingual (BA 17; $x=-6, y=-93, z=-4 \mathrm{~mm}$ ) gyri, and right lateral orbital frontal cortex (BA 47; $x=36, y=17$, $z=-13 \mathrm{~mm}$ ) and temporal pole BA $38 ; x=36, y=14$, $z=-23 \mathrm{~mm}$ ) activations identified in the comparison subjects (Table 2); and for the left hippocampus $(x=-38$, $y=-31, z=-7 \mathrm{~mm}$ ) and middle temporal (BA 39; $x=40$, $y=-68, z=29 \mathrm{~mm}$ ) gyrus deactivations observed in the gSAD subjects (Table 1 ).

Effect of treatment. All 12 gSAD subjects completed the 8-week flexible dose trial with nefazodone, however, one subject did not participate in a postreatment PET scan. Similar to the results of a prior open-label trial of nefazodone in patients with gSAD (VanAmeringen et al, 1999), nefazodone administration was associated with marked clinical improvement in symptoms of gSAD (Table 3). For the social anxiety imagery task, comparison of social anxiety-related neural activations prior to and after 8 weeks of nefazodone administration revealed greater activity in the left precentral gyrus, right insula, midbrain/ hypothalamus, and middle frontal and dorsal anterior cingulate cortex prior to treatment, and greater activity in the left middle occipital and bilateral lingual gyri, postcentral gyrus, gyrus rectus, and hippocampus after treatment (Table 4, Figure 3). For the confrontational mental arithmetic task, greater activity was observed in the right 
Table 2 Anatomical and Stereotaxic Location of Differential Neural Activations Related to Social Anxiety Provocation in Comparison Subjects

Coordinates $^{\mathrm{a}}$

\begin{tabular}{|c|c|c|c|c|}
\hline & & & & \\
\hline Brain region (Brodmann's area) & Voxel T & $x$ & $y$ & $\mathbf{z}$ \\
\hline Social anxiety > neutral imagery & & & & \\
\hline Inferior frontal gyrus $(R 47)^{b}$ & 4.97 & 36 & 17 & -13 \\
\hline Superior temporal gyrus $(38)^{\mathrm{b}}$ & 3.65 & 34 & 16 & -24 \\
\hline Lingual gyrus $(17)^{\mathrm{b}}$ & 4.06 & -6 & -90 & -6 \\
\hline Fusiform gyrus $(19)^{b}$ & 4.02 & 36 & -69 & -13 \\
\hline Ventral striatum $^{\mathrm{b}}$ & 3.36 & -20 & 2 & -8 \\
\hline Precuneus/posterior cingulate gyrus $(7 / 3 \mid$ ) & 3.28 & -14 & -53 & 32 \\
\hline Posterior STS (22) & 3.28 & 63 & -36 & II \\
\hline Medial frontal gyrus (9) & 3.25 & -2 & 50 & 34 \\
\hline Insula (anterior) & 3.11 & -34 & 15 & -4 \\
\hline
\end{tabular}

$\begin{array}{llrrr}\text { Mental arithmetic > neutral imagery } & & & & \\ \text { Posterior STS (R 21,22) } & 6.70 & 65 & -29 & \text { I } \\ \text { Thalamus } & 6.41 & -2 & -13 & 3 \\ \text { Inferior frontal gyrus (R 47) } & 5.93 & 34 & 19 & -14 \\ \text { Medial frontal gyrus (9) } & 5.68 & 10 & 44 & 16 \\ \text { Precuneus (7) } & 4.85 & -57 & -72 & 2 \\ \text { Inferior frontal gyrus (L 47) } & 4.50 & -30 & 13 & -11 \\ \text { Posterior STS (L 22) } & 4.05 & -59 & -57 & 21 \\ \text { Cerebellum } & 4.32 & 38 & -69 & -17 \\ \text { Middle frontal gyrus (6) } & 4.14 & 53 & 14 & 45\end{array}$

${ }^{a}$ Values represent the stereotaxic location of voxel maxima or minima with coordinates in Talairach space in millimeters lateral to midline $(x)$, anteriorl posterior to the anterior commissure $(y)$, and superior/inferior to the commissural line $(z)$. The $t$ statistics were uncorrected for multiple comparisons. ${ }^{b}$ Greater relative increase in activity associated with imagery-induced social anxiety in gSAD vs healthy comparison subjects.

Table 3 Effect of Nefazodone Pharmacotherapy on Symptoms and Disability Associated with Generalized Social Anxiety Disorder

\begin{tabular}{lcc}
\hline & \multicolumn{2}{c}{ Assessment } \\
\cline { 2 - 3 } Instrument & Baseline & End point \\
\hline LSAS total & $85.8 \pm 20.1$ & $40.0 \pm 29.7 *$ \\
Ham-A & $11.4 \pm 3.0$ & $7.1 \pm 3.0^{*}$ \\
Ham-D & $8.4 \pm 2.9$ & $6.1 \pm 4.8$ \\
FQ-Social & $21.4 \pm 6.1$ & $11.2 \pm 7.6^{*}$ \\
SDS-Social & $6.1 \pm 3.5$ & $1.9 \pm 1.9^{*}$ \\
SDS-Work & $4.4 \pm 3.1$ & $1.7 \pm 2.0^{*}$ \\
SDS-Home & $3.1 \pm 2.7$ & $1.0 \pm 1.5^{*}$ \\
\hline
\end{tabular}

LSAS total, the total of the subscale scores for the Leibowitz Social Anxiety Scale; HAM-A, Hamilton Anxiety Rating Scale; HAM-D, Hamilton Depression Rating Scale; FQ-Social, Social item subscale of the Fear Questionnaire; SDS, subscales of the Sheehan Disability Scale.

* $p<0.0$ I assesed using paired $t$-test.

inferior, bilateral middle and medial frontal cortex, midbrain/brainstem, and ventral anterior cingulate cortex prior to treatment, and greater activity in the visual cortex, inferior frontal and parietal cortex, brainstem, lingual gyrus, and orbital frontal cortex after treatment (Table 4, Figure 3).

The results of estimating a contrast between the pre- and post-treatment social anxiety imagery task effects and effects for a pretreatment LSAS scores of 90 and a posttreatment score of 20 or 45 indicated that treatment-related symptom remission and partial response are associated with both unique and common decreases and increases in social anxiety-related rCBF (Figure 4). Treatment-related decreases in rCBF that were unique $(p<0.005)$ to the remitted state included the ventral (BA 24; $x=-18$, $y=33, z=4 \mathrm{~mm}$ ) and dorsal (BA 32; $x=-8, y=12$, $z=40 \mathrm{~mm}$ ) anterior cingulate cortex, left ventrolateral (BA 45; $x=-51, y=25, z=4 \mathrm{~mm}$ ) and dorsolateral (BA 9; $x=-30, y=46, z=33 \mathrm{~mm}$ ) prefrontal cortex, and brainstem $(x=-8, y=-32, z=-19 \mathrm{~mm})$. Treatment-related increases in $\mathrm{rCBF}$ that were unique $(p<0.005)$ to the remitted state included the middle cingulate cortex (BA 24; $x=12, y=-14, z=39 \mathrm{~mm})$, left hippocampus/parahippocampal gyrus $(x=-36, y=-28, z=-10 \mathrm{~mm})$, subcallosal cortex (BA 25; $x=-4, y=23, z=-13 \mathrm{~mm}$ ), and the medial orbital (BA 11; $x=4, y=42, z=-16 \mathrm{~mm}$ ) and superior (BA 8; $x=18, y=46, z=44 \mathrm{~mm}$ ) frontal gyri. Decreases in social anxiety-related $\mathrm{rCBF}$ that were common to the remitted and partial response states included the lingual gyrus (BA 18; $x=8, y=-94, z=-14 \mathrm{~mm}$ and $x=8$, $y=-84, z=-16 \mathrm{~mm}$ ), left superior temporal gyrus (BA $22 ; x=-51, y=-11, z=8 \mathrm{~mm}$ and $x=-53, y=-11$, $z=13 \mathrm{~mm}$ ), and right ventrolateral frontal cortex (BA 45; $x=40, y=22, z=4 \mathrm{~mm}$ and $x=42, y=14, z=9 \mathrm{~mm})$. Increases in social anxiety-related $\mathrm{rCBF}$ that were common to the remitted and partial response states included the left middle occipital gyrus (BA 18; $x=-26, y=-85$, $z=3 \mathrm{~mm}$ and $x=-26, y=-85, z=3 \mathrm{~mm})$ and inferior parietal cortex (BA 40; $x=-24, y=-51, z=38 \mathrm{~mm}$ and $x=-24, y=-51, z=38 \mathrm{~mm})$.

\section{DISCUSSION}

Prior in vivo functional brain imaging studies of symptomatic states related to anxiety disorders have also utilized guided mental imagery to explore the neural response to mental re-enactment of anxiety-provoking situations (Shin et al, 1999; Britton et al, 2005). However, a concern was whether this approach to symptom provocation in individuals with gSAD would be countered by avoidance of anxious social situations, a major symptom cluster of SAD. This concern was addressed by a study design that incorporated an additional social anxiety condition in the form of a psychosocial stress task (ie confrontational mental arithmetic) for which unfamiliar others rather than the subjects themselves provoked anxiety states. This experimental condition also added ecological validity by assessing the response to core gSAD features of fear of actual social performance and evaluation. A serial subtraction task in combination with critical performance evaluation (Kirschbaum et al, 1993) was used. A similar task was recently used to demonstrate that gSAD is associated with an exaggerated stress response to a psychological stressor (Condren et al, 2002). 
Table 4 Anatomical and Stereotaxic Locations of Differences in Neural Activity Related to Re-Experiencing Social Anxiety-Provoking Situations (Left) or Social Performance and Evaluation (Mental Arithmetic, Right) in Subjects with Social Anxiety Disorder before vs after Nefazodone Pharmacotherapy

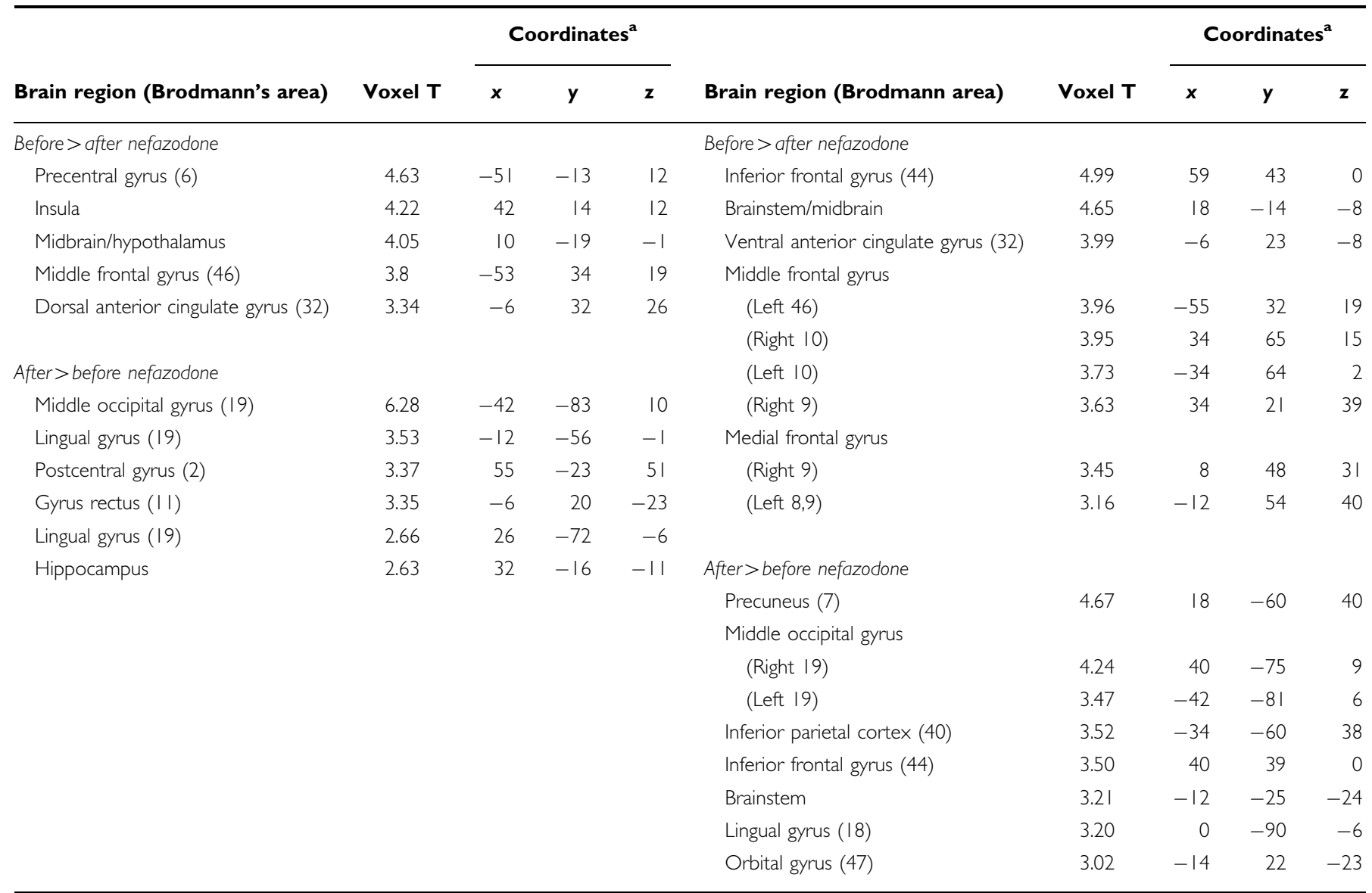

${ }^{a}$ Values represent the stereotaxic location of voxel maxima or minima with coordinates in Talairach space in millimeters lateral to midline (x), anterior/posterior to the anterior commissure $(y)$, and superior/inferior to the commissural line $(z)$.

The $t$ statistics were uncorrected for multiple comparisons.

Amygdala response to social anxiety. In vivo functional neuroimaging studies of healthy, nonpatient populations has generated a working model of the functional anatomy of physiological anxiety. Similar to those studies, the small sample of healthy volunteers studied here exhibited anxiety-related increases in activity in the ventrolateral prefrontal cortex and anterior temporal pole (Shin et al, 1999), and more widely in ventral prefrontal/limbic, dorsal prefrontal, and visual processing areas (Mataix-Cols et al, 2003) during the guided mental re-enactment of social situations associated with fear and avoidance. A comparison of social anxiety imagery-related neural activations in patients with gSAD and the comparison group indicated that gSAD was associated with distinct social anxiety-related neural activations, including decreases in amygdala activity.

Social anxiety induced by two distinct provocation approaches was in both instances associated with a decrease in amygdala activity in gSAD individuals. The observed decrease in social anxiety-related amygdala activity seemingly contradicts prior findings of exaggerated amygdala responses associated with SAD (Birbaumer et al, 1998; Stein et al, 2002; Schneider et al, 1999; Tillfors et al, 2001; Tillfors et al, 2002). However, a blunted amygdala response to anxiogenic stimuli was recently reported for individuals with PTSD (Britton et al, 2005) or spider phobias (Straube et al, 2006); combat veterans without PTSD exhibited a significant decrease in amygdala activity in response to traumatic/stressful mental imagery relative to combat veterans with PTSD (Britton et al, 2005). A decrease in amygdala activity when experiencing social anxiety may adaptively blunt emotional and autonomic responses when anxious, or decrease the cognitive salience of threat stimuli, and thus enhance overall functioning. This possibility is supported by the demonstration in animals that amygdala lesions attenuate contextual fear responses in rats (Baker and Kim, 2004) and emotional reactions to threat stimuli in monkeys (Izquierdo and Murray, 2004; Kalin et al, 2004). Moreover, recent human functional neuroimaging studies of the volitional suppression of responses to emotional stimuli (Ochsner et al, 2002, 2004) demonstrated that the cognitive suppression of responses to negative emotional stimuli was similarly associated with increased activity in the medial and lateral prefrontal cortex and decreased 
activity in the amygdala. Similar to the findings of Ochsner et al (2002), social anxiety-related activity in the left ventrolateral prefrontal cortex was inversely correlated with the right amygdala response (Figure 5). These findings
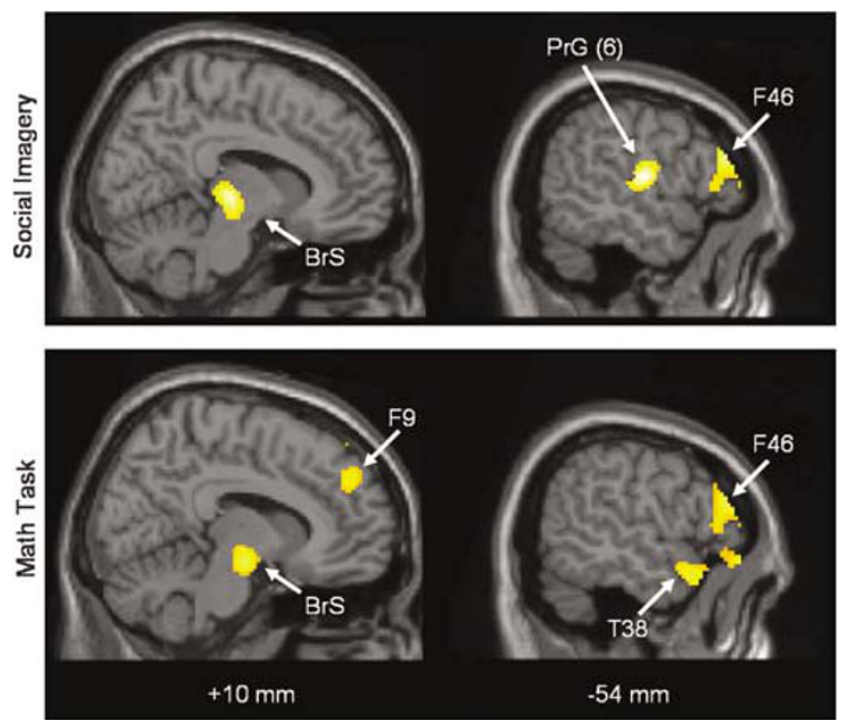

Figure 3 Greater $(p<0.005$, uncorrected) provoked social anxietyrelated rCBF before compared to after treatment of individuals with gSAD with nefazodone. Common effects are illustrated in parasagittal views for the social anxiety imagery (top) and confrontational mental arithmetic task provocation (bottom) conditions. Abbreviations refer to the right brainstem/midbrain (BrS), left middle (F46), and right medial (F9) frontal gyri, left precentral gyrus (PrG6), and left temporal pole (T38) suggest that gSAD is associated with an adaptive, top-down regulation of social emotion appraisal mechanisms by frontal cortical areas involved in cognitive control. The left ventrolateral prefrontal cortex has been similarly implicated in the suppression of habitual behavior; successful suppression was related to increasing activity in this brain region (Matsubara et al, 2004). In this study, the left ventrolateral prefrontal cortex response to the experience of social anxiety in individuals with gSAD was negatively correlated with their baseline LSAS scores and positively correlated with their self-rated anxiety response (Figure 5 , insets). The left ventrolateral prefrontal cortex may thus serve to mount an adaptive response to social anxiety-provoking situations that is compromised by gSAD in a graded relationship with gSAD symptom severity. Perhaps the central pathophysiology related to gSAD lies not in evaluative brain functions mediated by the amygdala and related brain regions, but rather in the failure of the left ventrolateral prefrontal cortex and related areas to cognitively regulate the response to socially anxious situations. The specific roles of the amygdala in the pathophysiology of SAD demands further investigation but the results of this study suggest that a simple model of SAD based on amygdala over-reactivity is clearly not warranted.

Effect of treatment of gSAD on the neural processing of social anxiety. The comparison of social anxietyrelated neural activations before and after a clinically efficacious course of nefazodone administration indicated that symptom improvement was associated with a decrease in social anxiety-related activity in the dorsolateral and

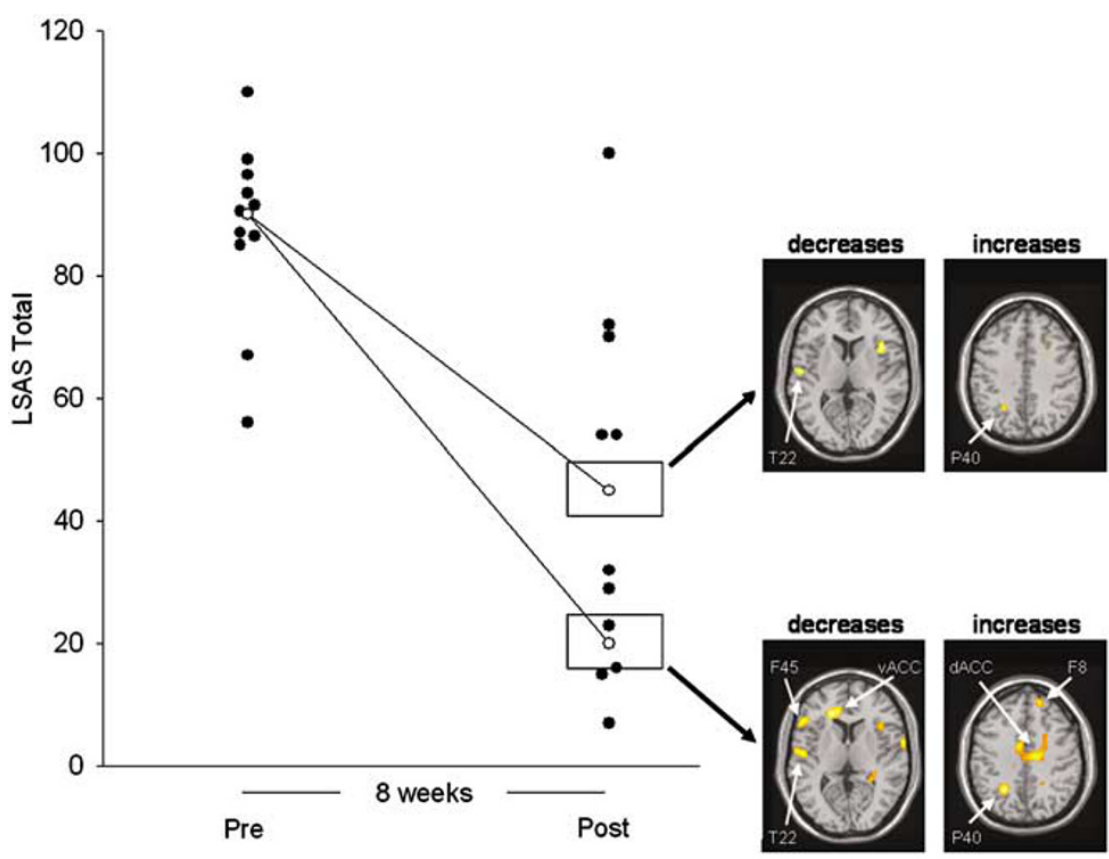

Figure 4 Distinct and common associations ( $p<0.005$, uncorrected) of social anxiety-related rCBF with symptom states reflecting either partial response and remission following treatment of individuals with gSAD with nefazodone. The left side of the figure illustrates the distribution of individual LSAS scores (total) prior to (pre-) and after (post-)treatment, with scores of 90, 45, and 20 (open circles) representing moderate to severe social anxiety disorder, partial response, and remission, respectively. Axial images on the right side of the figure illustrate distinct and common increases and decreases in rCBF (relative to the pretreatment baseline) for the partial response and remitted states. The social anxiety imagery condition was contrasted with the nonsocial imagery control condition for both treatment conditions. Abbreviations refer to left superior temporal gyrus (T22), ventral anterior cingulate cortex (vACC), inferior frontal gyrus (F45), dorsal anterior cingulate cortex (dACC), and frontal pole (F8). 

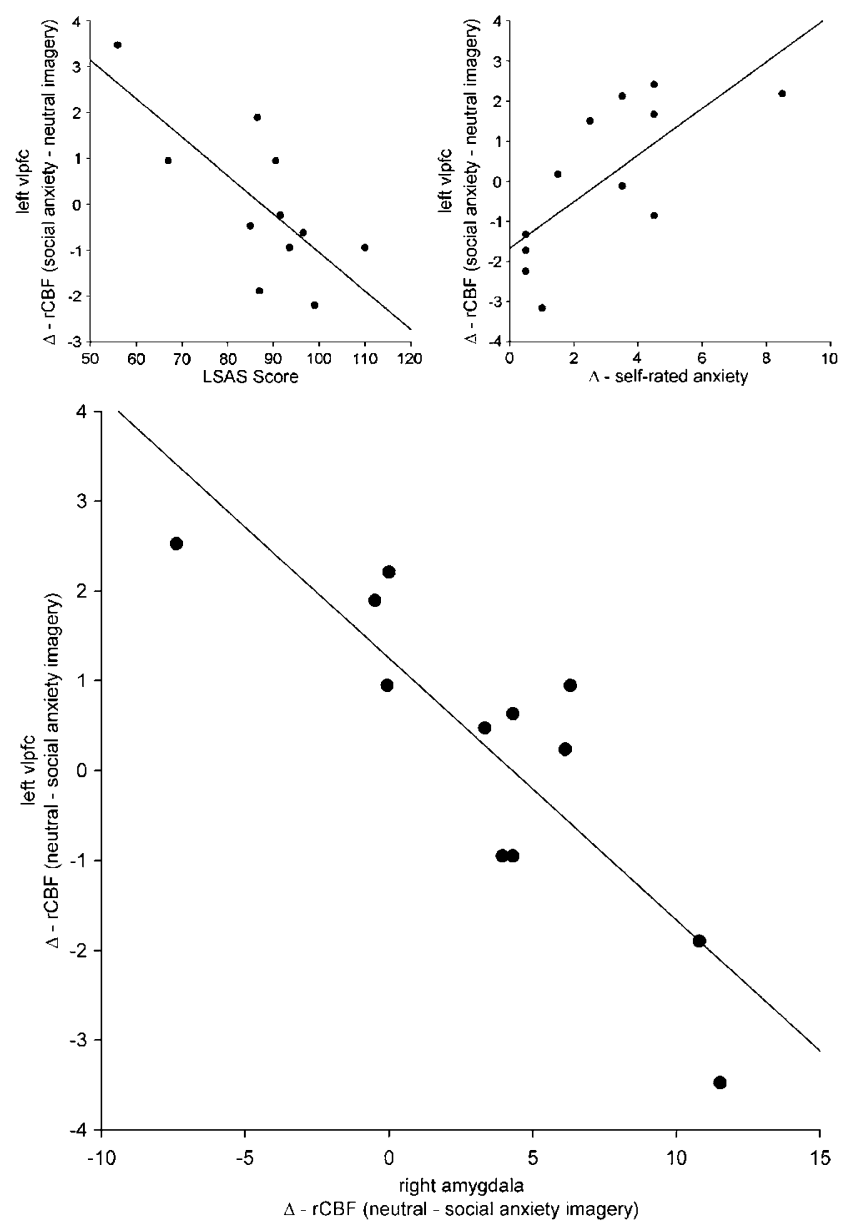

Figure 5 Negative correlation $(r=-0.85, p<0.004)$ between the left ventrolateral prefrontal cortex $(x=-48, y=44, z=-2 \mathrm{~mm})$ and right amygdala $(x=18, y=-4, z=-24 \mathrm{~mm})$ responses to the social anxiety vs neutral imagery conditions at the pretreatment assessment. The left ventrolateral prefrontal cortex response was also negatively correlated with gSAD symptom severity defined by the total LSAS score at the pretreatment assessment (left inset; $r=-0.74, p<0.0 \mathrm{I}$ ) and positively correlated with the self-rated anxiety response to social anxiety imagery (vs neutral imagery condition) at the pretreatment assessment (right inset; $r=0.72, p<0.03)$.

medial prefrontal cortex and dorsal anterior cingulate cortex, areas involved in cognitive control (Botvinick et al, 2001; Miller and Cohen, 2001) and self-reference (Kelley et al, 2002), and the midbrain/brainstem. These findings suggest that treatment of gSAD is associated with diminished cognitive representations of social anxiety such as diminished negative self-appraisal and negative rumination (Abbott and Rapee, 2004). Treatment-related decreases in social anxiety-related prefrontal activity were further assessed by a characterization and comparison of social anxiety-related neural responses associated with partial response and symptom remission (Figure 4). This analysis suggested that neocortical, limbic/paralimbic, and brainstem alterations in social anxiety-related activity are related to remission. Interestingly, altered activity in these brain regions has been previously implicated in depression remission following pharmacotherapy or psychotherapy (Mayberg et al, 2000; Goldapple et al, 2004). The left lateral prefrontal cortex responses associated with symptom remission closely resemble left ventrolateral and dorsolateral prefrontal cortex activations associated with expected threat-related distractors and inversely correlated with state anxiety in psychiatrically healthy individuals (Bishop et al, 2004), and their cognitive regulation (Ochsner et al, 2002 , 2004). The observed symptom remission-related decreases in lateral prefrontal cortex activity may thus additionally reflect a decreased demand for the engagement of cognitive control mechanisms thereby allocating information processing resources towards goal-related stimuli in the presence of salient threat-related distractors and improved functioning in the face of social anxiogenic situations.

Our statistical analysis was based on a mixed effects model that reflected aspects of the study design and was better suited for this study than a more naive GLM. By accounting for correlation among rCBF measurements from each individual's repeated scans, the mixed effects model borrowed strength from other scans in the series yielding less variable estimates of mean parameters. In turn, tests of hypotheses contrasting localized brain activity between conditions were more powerful than corresponding tests from a GLM.

Limitations of this study do, however, limit the strength and number of meaningful conclusions to be drawn from the study findings. Although neural responses were acquired for two distinct symptom provocation conditions, a clear question remains as to how well this PET study has captured the every day neural information processing abnormalities that characterize the symptoms of social anxiety in gSAD. Also, the events related to involvement of subjects in the PET imaging studies (ie head immobilized, vision field limited, subject of others attention) represents a social stressor in itself that was reflected by appreciable selfrated anxiety at rest. This nonzero baseline was modeled in the mixed effects model, but remains a challenge to pair-wise contrast of conditions approaches common to functional neuroimaging studies. The fixed order of this within-subject study of the unmedicated and medicated conditions poses a confound to defining treatment response. This order effect could have been estimated by a repeated measures design for the PET studies in the comparison subject group, though this design was not conducted here. The relatively small size of the comparison group also limits the power of directly assessing group differences related to the neural processing of social anxiety in attempts to define the neuropathophysiology of gSAD, though the power was sufficient to identify several important group differences in social anxiety-related neural processing. We refrain from going beyond functional inferences to make causal inferences about the relationship between nefazodone administration and alterations in distributed brain activity. Such statistical inferences require highly controlled experimental designs that are typically difficult to implement in functional neuroimaging studies and, in some cases, replicated empirical evidence across multiple studies. Instead, we limit our conclusions to identifying associations between statistically significant changes in measured $\mathrm{rCBF}$ and nefazodone administration. Finally, the degree to which the results of the comparison of provoked symptom states prior to and after a regimen of nefazodone administration are unique to the 
mechanistically novel actions of nefazodone (Davis et al, 1997) or generalize to treatment response to other medication or psychotherapy approaches is unknown.

In summary, this PET imaging study used novel study designs and image analysis techniques to add additional knowledge to the now many attempts to define the neural basis of pathological social anxiety states associated with $\mathrm{SAD}$, and the less studied basis of response to treatment. The new knowledge is highlighted by the unconventional observation that decreases, rather than increases, in right amygdala activity are associated with symptomatic states, perhaps reflecting adaptive mechanisms of buffering anxiety responses and improving functioning. Treatment response was associated with diminished prefrontal cortical responses to symptomatic states, perhaps reflecting decreased ruminations about anxiogenic stimuli (Abbott and Rapee, 2004) or a lessened demand for cognitive regulation of responses to such stimuli. The contention that symptom remission and partial response are related to distinct neural responses to treatment (Mayberg et al, 2000; Goldapple et al, 2004) is also supported by these findings.

\section{ACKNOWLEDGEMENTS}

This study was supported by an investigator-initiated grant from Bristol-Myers Squibb Pharmaceuticals and by the Emory Conte Center for the Psychobiology of Major Mental Disorders (NIH MH58922). Partial support was also provided by $\mathrm{NIH}$ via K25 MH65473. The authors gratefully acknowledge the contributions of Lisa Page and Margie Jones. The generous commitment of the individuals with social anxiety disorder to participate as research volunteers is also gratefully acknowledged.

\section{REFERENCES}

Abbott MJ, Rapee RM (2004). Post-event rumination and selfappraisal in social phobia before and after treatment. J Abnorm Psychol 113: 136-144.

Adolphs R (2001). The neurobiology of social cognition. Curr Opin Neurobiol 11: 231-239.

Baker KB, Kim JJ (2004). Amygdalar lateralization in fear conditioning: evidence for greater involvement of the right amygdala. Behav Neurosci 118: 15-23.

Bell CJ, Malizia AL, Nutt DJ (1999). The neurobiology of social phobia. Eur Arch Psychiatr Clin Neurosci 249: S11-S18.

Birbaumer N, Grodd W, Diedrich O, Klose U, Erb M, Lotze M et al (1998). fMRI reveals amygdala activation to human faces in social phobics. Neuroreport 9: 1223-1226.

Bishop S, Duncan J, Brett M, Lawrence AD (2004). Prefrontal cortical function and anxiety: controlling attention to threatrelated stimuli. Nat Neurosci 7: 184-188.

Botvinick M, Braver TS, Carter CS, Cohen JD (2001). Conflict monitoring and cognitive control. Psychol Rev 108: 624-652.

Bourne EJ (1995). The Anxiety and Phobia Workbook, 2nd edn. New Harbinger Publications, Inc.: Oakland, CA.

Bowman D, Kilts CD (2003). Modeling intra-subject correlation among repeated scans in positron emission tomography (PET) neuroimaging data. Hum Brain Mapp 20: 59-70.

Breiter HC, Rauch SL, Kwong KK, Baker JR, Weisskoff RM, Kennedy DN et al (1996). Functional magnetic resonance imaging of symptom provocation in obsessive-compulsive disorder. Arch Gen Psychiatr 53: 595-606.

Britton JC, Phan KL, Taylor SF, Fig LM, Liberzon I (2005). Corticolimbic blood flow in posttraumatic stress disorder during script-driven imagery. Biol Psychiatr 57: 832-840.

Brothers L (1990). The social brain: a project for integrating primate behavior and neurophysiology in a new domain. Concepts Neurosci 1: 27-51.

Chartier MJ, Hazen AL, Stein MB (1998). Lifetime patterns of social phobia: a retrospective study of the course of social phobia in a nonclinical population. Depression Anxiety 7: 113-121.

Condren RM, O'Neill A, Ryan MCM, Barrett P, Thakore JH (2002). HPA axis response to a psychological stressor in generalised social phobia. Psychoneuroendocrinol 27: 693-703.

Cox BJ, Fleet C, Stein MB (2004). Self-criticism and social phobia in the US national comorbidity survey. J Affect Disord 82: 227-234.

Davidson JT, Hughes DL, Georges LK (1993). The epidemiology of social phobia: findings from the Duke Epidemiological Catchment Area Study. Psychol Med 23: 709-718.

Davis R, Whittington X, Bryson HM (1997). Nefazodone - a review of its pharmacology and clinical efficacy in the management of major depression. Drugs 53: 608-636.

den Boer JA (1997). Social phobia: epidemiology, recognition, and treatment. $B M J$ 315: 796-800.

First MB, Spitzer RL, Robert L, Gibbon M, Williams JBW (2002). Structured Clinical Interview for DSM-IV-TR Axis I Disorders, Research Version, Patient Edition. American Psychiatric Press: Washington, DC.

Furmark T, Tillfors M, Marteinsdottir I, Fischer H, Pissiotta A, Langstrom B et al (2002). Common changes in cerebral blood flow in patients with social phobia treated with citalopram or cognitive-behavioral therapy. Arch Gen Psychiatr 59: 425-433.

Goldapple K, Segal Z, Garson C, Lau M, Bieling P, Kennedy S et al (2004). Modulation of cortical-limbic pathways in major depression: treatment-specific effects of cognitive behavioral therapy. Arch Gen Psychiatr 61: 34-41.

Hamilton M (1960). Diagnosis and rating scale for depression. Br J Psychiatr 3: 76-79.

Izquierdo A, Murray EA (2004). Combined unilateral lesions of the amygdala and orbital frontal cortex impair affective processing in rhesus monkeys. J Neurophysiol 91: 2023-2039.

Kalin NH, Shelton SE, Davidson RJ (2004). The role of the central nucleus of the amygdala in mediating fear and anxiety in the primate. J Neurosci 24: 5506-5515.

Katzelnick DJ, Kobak KA, DeLeire T, Henk HJ, Greist JH, Davidson J et al (2001). Impact of generalized social anxiety disorder in managed care. Am J Psychiatr 158: 1999-2007.

Kelley WM, Macrae CN, Wyland CL, Caglar S, Inati S, Heatherton TF (2002). Finding the self: An event-related fMRI study. J Cognit Neurosci 14: 785-794.

Kessler RC, Berglund P, Demler O, Jin R, Walters EE (2005a). Lifetime prevalence and age-on-onset distributions of DSM-IV disorders in the national comorbidity survey replication. Arch Gen Psychiatr 62: 593-602.

Kessler RC, Chiu WT, Demler O, Walters EE (2005b). Prevalence, severity, and comorbidity of 12-month DSM-IV disorders in the national comorbidity survey replication. Arch Gen Psychiatr 62: 617-627.

Kessler RC, Stein MB, Berglund PA (1998). Social phobia subtypes in the National Comorbidity Survey. Am J Psychiatr 155: 613-619.

Kimbrell TA, George MS, Parekh PI, Ketter TA, Podell DM, Danielson AL et al (1999). Regional brain activity during transient self-induced anxiety and anger in healthy adults. Biol Psychiatr 46: 454-465. 
Kirschbaum C, Pirke KM, Hellhammer DH (1993). The Trier Social Stress Test: a tool for investigating psychobiological stress responses in a laboratory setting. Neuropsychoendocrinology 28: 76-81.

Liebowitz MR (1987). Social phobia. In: Klein DF (ed). Modern Problems in Pharmacopsychiatry: Anxiety, Vol. 22. Karger: Basel, NY. pp 141-173.

Liebowitz MR, Gorman JM, Fyer AJ, Klein DF (1985). Social phobia: review of a neglected anxiety disorder. Arch Gen Psychiatr 42: 729-736.

Mataix-Cols D, Cullen S, Lange K, Zelaya F, Andrew C, Amaro E et al (2003). Neural correlates of anxiety associated with obsessive-compulsive symptom dimensions in normal volunteers. Biol Psychiatr 53: 482-493.

Mathew SJ, Coplan JD, Gorman JM (2001). Neurobiological mechanisms of social anxiety disorder. Am J Psychiatr 158: 1558-1567.

Matsubara M, Yamaguchi S, Xu J, Kobayashi S (2004). Neural correlates for the suppression of habitual behavior: a functional MRI study. J Cogn Neurosci 16: 944-954.

Mayberg HS, Branna SK, Tekell JL, Silva JA, Mahurin RK, McGinnis S et al (2000). Regional metabolic effects of fluoxetine in major depression: serial changes and relationship to clinical response. Biol Psychiatr 48: 830-843.

Mazziotta JC, Huang SC, Phelps ME (1985). A noninvasive positron computed tomography technique using oxygen-15labeled water for the evaluation of neurobehavioral task batteries. J Cereb Blood Flow Metab 5: 70-78.

Miller EK, Cohen JD (2001). An integrative theory of prefrontal cortex function. Ann Rev Neurosci 24: 167-202.

Neter J, Kutner MH, Nachtsheim CJ, Wasserman W (1996). Applied Linear Statistical Models, 4th edn. McGraw Hill/Irwin: Chicago, IL.

Ochsner KN, Bunge SA, Gross JJ, Gabrieli JDE (2002). Rethinking feelings: an fMRI study of the cognitive regulation of emotion. J Cogn Neurosci 14: 1215-1229.

Ochsner KN, Ray RD, Cooper JC, Robertson ER, Chopra S, Gabrieli JDE et al (2004). For better or for worse: neural systems supporting the cognitive down- and up-regulation of negative emotion. NeuroImage 23: 483-499.

Pitman RK, Orr SP, Forgue DF, de Jong JB, Claiborn JM (1987). Psychophysiologic assessment of posttraumatic stress disorder imagery in Vietnam combat veterans. Arch Gen Psychiatry 44: 970-975.

Rapee RM, Heimberg RG (1997). A cognitive-behavioral model of anxiety in social phobia. Behav Res Ther 35: 741-756.

Rauch SL, Savage CR, Alpert NM, Miguel EC, Baer L, Breiter HC et al (1995). A positron emission tomography study of simple phobic symptom provocation. Arch Gen Psychiatr 52: $20-28$.

Safren SA, Heimberg RG, Brown EJ, Holle C (1997). Quality of life in social phobia. Anxiety 4: 126-133.

Saxena S, Brody AL, Maidment KM, Dunkin JJ, Colgan M, Alborzian S et al (1999). Localized orbitofrontal and subcortical metabolic changes and predictors of response to paroxetine treatment in obsessive-compulsive disorder. Neuropsychopharmacology 21: 683-693.

Schneider F, Weiss U, Kessler C, Müler-Gärtner HW, Poss S, Salloum JB et al (1999). Subcortical correlates of differential classical conditioning of aversive emotional reactions in social phobia. Biol Psychiatr 45: 863-871.

Schneier FR, Heckelman LR, Garfinkel R, Campeas R, Fallon BA, Gitow A et al (1994). Functional impairment in social phobia. $J$ Clin Psychiatr 55: 322-331.

Schneier FR, Johnson J, Hornig CD, Liebowitz MR, Weissman MM (1992). Social phobia: comorbidity and morbidity in an epidemiologic sample. Arch Gen Psychiatr 49: 282-288.

Schneier FR, Liebowitz MR, Abi-Dargham A, Zea-Ponce Y, Lin SH, Laruelle $M(2000)$. Low dopamine $\mathrm{D}_{2}$ receptor binding potential in social phobia. Am J Psychiatr 157: 457-459.

Sheehan PQ (1967). A shortened form of Bett's questionnaire on mental imagery. J Clin Psychol 223: 380-389.

Shin LM, McNally RJ, Kosslyn SM, Thompson WL, Rauch SL, Alpert NM et al (1999). Regional cerebral blood flow during script-driven imagery in childhood sexual abuse-related PTSD: a PET investigation. Am J Psychiatr 156: 575-584.

Stein MB, Goldin PR, Sareen J, Zorrilla L, Brown GG (2002). Increased amygdala activation to angry and contempuous faces in generalized social phobia. Arch Gen Psychiatr 59: 1027-1034.

Stein MB, Kean YM (2000). Disability and quality of life in social phobia: epidemiologic findings. Am J Psychiatr 157: 16061613.

Stein MB, Walker JR, Forde DR (1996). Public speaking fears in a community sample: prevalence, impact on functioning, and diagnostic classification. Arch Gen Psychiatr 53: 169-174.

Straube T, Glauer M, Dilger S, Mentzel HJ, Miltner WHR (2006). Effects of cognitive-behavioral therapy on brain activation in specific phobia. NeuroImage 29: 125-135.

Talairach J, Tournoux P (1988). Co-Planar Stereotaxic Atlas of the Human Brain. Thieme Medical Publishers: New York. $122 \mathrm{pp}$.

Tiihonen J, Kuikka J, Bergstrom K, Lepola U, Koponen H, Leinonen E (1997). Dopamine reuptake site densities in patients with social phobia. Am J Psychiatr 154: 239-242.

Tillfors M, Furmark T, Marteinsdottir I, Fredrickson M (2002). Cerebral blood flow during anticipation of public speaking in social phobia: a PET study. Biol Psychiatr 52: 1113-1119.

Tillfors M, Furmark T, Marteinsdottir I, Fischer H, Pissiota A, Landstrom B et al (2001). Cerebral Blood Flow in Subjects With Social Phobia During Stressful Speaking Tasks: A PET Study. Am J Psychiatr 158: 1220-1226.

Verbeke G, Molenberghs G (2000). Linear Mixed Models for Longitudinal Data. Springer: New York.

Weiller E, Bisserbe JC, Boyer P, Lepine JP, Lecrubier Y (1996). Social phobia in general health care: an unrecognised undertreated disabling disorder. Br J Psychiatr 168: 169-174.

Wittchen HU, Fuetsch M, Sonntag H, Muller N, Liebowitz M (2000). Disability and quality of life in pure and comorbid social phobia. Findings from a controlled study. Eur Psychiatr 15: 46-58.

Woods RP, Grafton ST, Holmes CJ, Cherry SR, Mazziotta JC (1998a). Automated image registration: I. General methods and intrasubject, intramodality validation. J Comput Assist Tomogr 22: $139-152$.

Woods RP, Grafton ST, Watson JDG, Sicotte NL, Mazziotta JC (1998b). Automated image registration: II. Intersubject validation of linear and nonlinear models. J Comput Assist Tomogr 22: 53-165. 